\title{
ON SCHREIER VARIETIES OF LINEAR ALGEBRAS
}

\author{
BY \\ JACQUES LEWIN
}

I. Introduction. A variety $\mathfrak{v}$ of algebraic systems (i.e., a class of algebras defined by identical relations) in which the subalgebras of free algebras are again free is called a Schreier variety. Thus the varieties of groups (Schreier [8]), abelian groups, vector spaces, linear (nonassociative) algebras (Kuroš [5]), Lie algebras (Šršov [9], Witt [12]), commutative algebras, anticommutative algebras (Šršov [10]) are Schreier varieties. Some of the varieties are also what we may call Nielsen varieties, i.e., if $g_{1}, \ldots, g_{n}$ generate the subalgebra $G$ of the free $\mathfrak{v}$-algebra $F$, then there is an effective procedure for obtaining a free set of generators for $G$. Thus the varieties of abelian groups, vector spaces, groups (Nielsen [7]), Lie algebras (Cohn [2]) are Nielsen varieties. The main purpose of this paper is to show (§III and §IV) that a variety of linear algebras over an infinite field is a Schreier variety if and only if it is a Nielsen variety. The main step of the proof is to show that one can "reduce" the elements $g_{1}, \ldots, g_{n}$ of a free algebra in a Schreier variety to a free set of generators by applying a sequence of "elementary transformations" i.e., transformations which (a) replace a sequence $s_{1}, \ldots, s_{n}$ by a linear transform of $s_{1}, \ldots, s_{n}$ or (b) fix $s_{1}, \ldots, s_{n-1}$ and replace $s_{n}$ by $s_{n}+w$, with $w$ in the algebra generated by $s_{1}, \ldots, s_{n-1}$.

Our main theorem then enables us to find a set of generators for the automorphism group of a finitely generated free algebra in a Schreier variety (see Cohn [2] for the case of Lie algebras) and to solve the generalized word problem for the free algebras in Schreier varieties defined by finitely many multilinear identical relations $(\S \mathrm{V})$. Our results apply in particular to the varieties of all linear algebras, commutative algebras, anticommutative algebras, and Lie algebras.

\section{Notation and preliminary results.}

1. All algebras are not necessarily associative linear algebras with identity 1 over the infinite field $\phi$. If $S$ is a subset of an algebra $A,\langle S\rangle, \operatorname{Alg}(S), \operatorname{Id}(S)$ denote respectively the subspace, subalgebra, ideal of $A$ generated by $S$.

Let $X$ be a set. We denote by $F=F(X)$ the free algebra freely generated by $X$. Thus if $M=M(X)$ is the free groupoid (see e.g., [1, p. 1]) on $X$, then $M \cup\{1\}$ is a $\phi$-basis for $F$, and the multiplication in $F$ is an extension by linearity of the multiplication in $M \cup\{1\}$. A monomial in $F$ is an element of $M \cup\{1\}$. The degree $d(m)$ of a monomial is defined as usual by setting $d(1)=0, d(x)=1$ for $x \in X$ and $d(x m)=d(m x)=d(m)+1$ for $x \in X$ and $m \in M$. Let $H^{k}$ be the subspace of

Received by the editors April 14, 1967. 
$F$ generated by the monomials of degree $k$. Then $F$ is graded by the $H^{i}$ i.e., $F=$ $\sum_{i=0}^{\infty} \oplus H^{i}$ and $H^{i} H^{j} \subset H^{i+j}$. An element of $F$ is homogeneous if it lies in some $H^{i}$.

Let now $N$ be an ideal of $F$ generated by a set of homogeneous elements and let $\eta: F \rightarrow F / N=A$ be the natural epimorphism. Then it is easily verified that the $H^{i} \eta$ grade $A$. If $m$ is a monomial in $F$, we also call $m \eta$ a monomial. It is clear that $H^{i} \eta$ has a basis consisting of monomials, and thus so does $A$. We choose a fixed monomial basis $B$ for $A$. Then, if $0 \neq w \in A, w$ can be uniquely written as

$$
w=\sum_{i=1}^{n} \alpha_{i} m_{i}, \quad \alpha_{i} \neq 0, m_{i} \in B, m_{i}=m, \Leftrightarrow i=j .
$$

We call $n$ the length of $w$. Likewise, since the $H^{i} \eta$ generate their direct sum, $w$ can also be uniquely written as

$$
w=\sum_{i=1}^{l} h_{k_{i}}, \quad h_{k_{i}} \neq 0, h_{k_{i}} \in H^{k_{i}}, k_{1}<k_{2}<\cdots<k_{l} .
$$

We call the $h_{k_{i}}$ 's the homogeneous terms of $w$ and we call $l$ the homogeneous length of $w . w$ is homogeneous if it has homogeneous length $1 . d(w)=k_{l}$ is the degree of $w$, and $i(w)=k_{1}$ is the index of $w$. It is clear that if $h_{1}$ and $h_{2}$ are homogeneous and $h_{1} h_{2} \neq 0$, then $d\left(h_{1} h_{2}\right)=d\left(h_{1}\right)+d\left(h_{2}\right)$. Also, if $w_{1}$ and $w_{2}$ are distinct elements of $A$, then $d\left(w_{1}-w_{2}\right) \leqq \max \left(d\left(w_{1}\right), d\left(w_{2}\right)\right)$ and $i\left(w_{1}-w_{2}\right) \geqq \min \left(i\left(w_{1}\right), i\left(w_{2}\right)\right)$.

If $S$ is a finite subset of $A$, the degree (homogeneous length) of $A$ is the sum of the degrees (homogeneous lengths) of the nonzero elements of $S$.

We will make repeated use of the following (probably well known) fact:

Proposition 1. Let $A$ be a finitely generated graded algebra as defined above. If $\theta$ is an endomorphism of $A$ onto $A$, then $\theta$ is an automorphism.

Proof. Suppose $w \in \operatorname{Ker} \theta$, with $d(w)=d$. Let $K=\sum_{i=d+1}^{\infty} \oplus H^{i} \eta$. Then $K$ is an ideal of $A$ and $w \notin K$. Since $K \theta \subset K, \theta$ induces an endomorphism $\dot{\theta}$ of $A / K$ onto $A / K$, and since $A / K$ is finite dimensional, $\bar{\theta}$ is an automorphism. However, $(w+K) \bar{\theta}$ $=w \theta+K=K$ and hence $w \in K$. This contradiction proves the proposition.

2. Let now $X$ be countably infinite and let $V$ be a subset of $F(X)$. If $A$ is an algebra we define $\mathfrak{v}(A)$ to be the ideal of $A$ generated by all the elements $v\left(a_{1}, \ldots, a_{k}\right)$ where $v\left(x_{1}, \ldots, x_{k}\right) \in V, x_{i} \in X$ and $a_{i} \in A$. The variety $\mathfrak{v}$ is the class of all those algebras $A$ for which $\mathfrak{v}(A)=0$. Let $Y$ be a set of cardinal $c$. The algebra $F(\mathfrak{v}, Y)$ $=F(Y) / \mathfrak{v}(F(Y))$ is called the free $\mathfrak{v}$-algebra of rank $c$ freely generated by $Y$. An algebra $A$ is a free $\mathfrak{v}$ algebra if it is isomorphic to $F(\mathfrak{v}, Y)$ for some $Y$. A subset $S$ of an algebra in $v$ is a $\mathfrak{v}$-free set if it freely generates a free $\mathfrak{v}$-algebra. Thus $S$ is a $\mathfrak{v}$-free set if whenever $u\left(s_{1}, \ldots, s_{k}\right)=0$, with $s_{1}, \ldots, s_{k} \in S$, then $u\left(x_{1}, \ldots, x_{k}\right) \in \mathfrak{v}(F(X))$. We note that the above are all standard notions in the theory of universal algeb ras (see e.g., [3]).

Since $\phi$ is assumed to be infinite, we may apply a familiar Vandermonde determinant argument (see e.g., [4, Proposition 2, p. 234]) to prove the crucial 
Proposition 2. If $Y$ is any set, and $V$ any subset of $X$, then $\mathfrak{v}(F(Y))$ is generated by a set of homogeneous elements.

Thus the notions of $\S I$ are applicable to $F(\mathfrak{v}, Y)$.

A variety is a Schreier variety if the subalgebras of free $\mathfrak{b}$-algebras are again free $\mathfrak{v}$-algebras.

3. Let now $F\left(\mathfrak{v},\left\{x_{1}, \ldots, x_{n}\right\}\right)$ be the free algebra of rank $n$ in some variety $\mathfrak{v}$. A transformation $\tau:\left(x_{1}, \ldots, x_{n}\right) \rightarrow\left(y_{1}, \ldots, y_{n}\right)$ is an elementary transformation of order $n$ if either

(i) $\tau$ is a nonsingular $\phi$-linear transformation, or

(ii) $y_{1}=x_{1}, \ldots, y_{n-1}=x_{n-1}, y_{n}=x_{n}+w$ with $w \in \operatorname{Alg}\left(x_{1}, \ldots, x_{n-1}\right)$.

Let $A$ be any algebra in $\mathfrak{v}$ and $\left(a_{1}, \ldots, a_{n}\right)$ an $n$-tuple of elements of $A$. Let also $\varphi: F \rightarrow A$ be the homomorphism for which $x_{i} \varphi=a_{i}$. We define $\left(a_{1}, \ldots, a_{n}\right) \tau$ by setting $\left(a_{1}, \ldots, a_{n}\right) \tau=\left(b_{1}, \ldots, b_{n}\right)$ with $b_{i}=y_{i} \varphi$.

$\mathfrak{v}$ is a Nielsen variety if whenever $a_{1}, \ldots, a_{m}$ are elements of a free $\mathfrak{v}$ algebra $F$ of finite rank, then there is a sequence $\tau_{1}, \ldots, \tau_{k}$ of elementary transformations of order $m$ such that

$$
\left(\cdots\left(\left(a_{1}, \ldots, a_{m}\right) \tau_{1}\right) \cdots\right) \tau_{k}=\left(b_{1}, \ldots, b_{m}\right)
$$

and the terms of positive degree among $b_{1}, \ldots, b_{m}$ freely generate the free subalgebra $\operatorname{Alg}\left(a_{1}, \ldots, a_{m}\right)$ of $F$.

\section{Schreier varieties are Nielsen varieties.}

1. Let $F=F(\mathfrak{v}, X)$ be the free $\mathfrak{v}$-algebra of countable rank freely generated by $X$ in some variety $\mathfrak{v}$. We now drop the $\mathfrak{v}$ from $\mathfrak{v}$-algebra, $\mathfrak{v}$-free, etc., whenever the context is clear. A subalgebra $R$ of $F$ is homogeneous if it has a generating set which consists of homogeneous elements. $R$ is homogeneous if, and only if, whenever $w \in R$ then the homogeneous terms of $w$ are also in $R$.

We first consider homogeneous subalgebras.

LEMMA 1. Let $R$ be a homogeneous subalgebra of $F$ and suppose $R$ is generated by $Y=\left\{y_{1}, \ldots, y_{n}\right\}$. Let $h_{i}$ be the homogeneous term of $y_{i}$ of highest degree. If $H=$ $\left\{h_{1}, \ldots, h_{n}\right\}$ is a free set, then $H$ freely generates $R$.

Proof. We proceed by induction on the homogeneous length of $Y$. If the homogeneous length of $Y$ is $n$, then either each $y_{i}$ is homogeneous or some $y_{i}$ is a constant and there is nothing to prove. Suppose then that the lemma has been proved for sets of generators of homogeneous length $s$, and that $Y$ has homogeneous length $s+1$.

We reorder the $y_{i}$ 's if necessary so that $y_{1}=h_{1}, \ldots, y_{r}=h_{r}$ are homogeneous, and

$$
y_{r+i}=l_{r+i}+\bar{y}_{r+i}+h_{r+i}, \quad i=1, \ldots, n-r,
$$

with $l_{r+i}$ the homogeneous term of least degree in $y_{r+i}$. We may further assume that 
$d\left(l_{r+i}\right) \leqq d\left(l_{r+i+1}\right)$. Since $R$ is homogeneous, $l_{r+1} \in R$ and hence there is an element

$$
w\left(x_{1}, \ldots, x_{n}\right)=\sum_{i=1}^{k} \alpha_{i} m_{i}\left(x_{1}, \ldots, x_{n}\right), \quad m_{i} \in B, \alpha_{i} \neq 0
$$

of $F$, which we may choose to be of minimal length, for which

$$
l_{r+1}=w\left(y_{1}, \ldots, y_{n}\right) \text {. }
$$

It is clear that if $m$ is a monomial, then either $m\left(h_{1}, \ldots, h_{n}\right)=0$ or $d\left(m\left(h_{1}, \ldots, h_{n}\right)\right)$ $=d\left(m\left(y_{1}, \ldots, y_{n}\right)\right)$. Now, if $m_{l}\left(h_{1}, \ldots, h_{r}\right)=0$ for some $l$, then, since $H$ is a free set, $m_{l}\left(y_{1}, \ldots, y_{n}\right)=0$, and hence $l_{r+1}=\sum_{i \neq l} \alpha_{i} m_{i}\left(y_{1}, \ldots, y_{n}\right)$, a contradiction to the minimality of the length of $w$. We may then partition the set $\{1, \ldots, k\}$ by declaring that $j \in S_{1}$, if $m_{j}\left(h_{1}, \ldots, h_{n}\right)=\max _{1 \leqq j \leqq r} d\left(m_{j}\left(h_{1}, \ldots, h_{n}\right)\right)$ and $j \in S_{2}$ otherwise. If we put $h=\sum_{i \in S_{1}} \alpha_{i} m_{i}\left(h_{1}, \ldots, h_{n}\right)$, then equating terms of the same degree in (1), we see that $h=0$ or $h=l_{r+1}$. If $h=0$, then $\sum_{i \in S_{1}} \alpha_{i} m_{i}\left(y_{1}, \ldots, y_{n}\right)=0$ and we again contradict the minimality of $w$. Thus $h=l_{r+1}$. But then, since $d\left(h_{r+i}\right)>d\left(l_{r+1}\right)$, each $m_{i}$ has degree 0 or is monomial in $x_{1}, \ldots, x_{r}$ only. Thus $w\left(y_{1}, \ldots, y_{n}\right) \in \operatorname{Alg}\left(y_{1}, \ldots, y_{r}\right)$ and the set $Y^{\prime}=\left\{y_{1}^{\prime}, \ldots, y_{n}^{\prime}\right\}$ defined by

$$
y_{1}^{\prime}=y_{1}, \ldots, y_{r}^{\prime}=y_{r}, \quad y_{r+1}^{\prime}-w\left(y_{1}, \ldots, y_{n}\right), \quad y_{r+2}^{\prime}=y_{r+2}, \ldots, y_{n}^{\prime}=y_{n}
$$

is again a set of generators for $R$. However, $Y^{\prime}$ has homogeneous length $s$, and the homogeneous term of $y_{1}^{\prime}$ of highest degree is still $h_{i}$. Thus, applying the induction hypothesis, $R=\operatorname{Alg}(H)$ and the lemma is proved.

Let $F^{k}=\sum_{i=0}^{k} H^{k}$. If $R$ is a subalgebra of $F$, we set, following Witt [11], $R_{-1}=0$, $R_{k}=R \cap F^{k}$ and $R_{k}^{\prime}=\operatorname{Alg}\left(R_{k-1}\right) \cap F^{k}$. We can now easily prove

LEMMA 2. Let $H=\left\{h_{1}, \ldots, h_{n}\right\}$ be a set of homogeneous elements of $F$. Let $H_{k}=\left\{h_{\imath} \mid d\left(h_{i}\right)=k\right\}$ and let $R=\operatorname{Alg}(H)$. Then $\left\langle H_{k}\right\rangle=R_{k} \bmod R_{k}^{\prime}$ and $\operatorname{Alg}\left(R_{k}\right)$ $=\mathrm{Alg}\left(H_{1} \cup \cdots \cup H_{k}\right)$.

We can now prove our main theorem for homogeneous subalgebras.

THEOREM 1. Let $\mathfrak{v}$ be a variety in which finitely generated homogeneous subalgebras of free $\mathfrak{v}$-algebras are again free $\mathfrak{v}$-algebras. Let $H=\left\{h_{1}, \ldots, h_{n}\right\}$ be a set of homogeneous elements of $F$ and let $R=\operatorname{Alg}(H)$. Then some subset of $H$ freely generates $R$.

Proof. The theorem is obvious if $n=1$. Suppose then, for an induction, that we have proved the theorem for sets of at most $n-1$ elements.

Let $L=\left\{h_{1}, \ldots, h_{n-1}\right\}$. If $L$ is not free then, by the induction hypothesis some proper subset $L^{\prime} \subset L$ generates $\operatorname{Alg}(L)$. But then, $R=\operatorname{Alg}\left(L^{\prime}, h_{n}\right)$ is generated by at most $n-1$ elements and we may again use the induction hypothesis to prove the theorem. Thus we may assume that $R$ has rank at least $n-1$. However, if $R$ has rank $n$, then, by Proposition $1, H$ is a free set and there is nothing to prove. Thus we may assume that $R$ has rank $n-1$. 
Our first task is to show that $R$ has a set of homogeneous free generators. To this effect, let $Y=\left\{y_{1}, \ldots, y_{n-1}\right\}$ be a set of free generators of $R$ with homogeneous terms of highest degree $z_{1}, \ldots, z_{n-1}$ such that the degree of $Y$ is minimal. If $Z$ $=\left\{z_{1}, \ldots, z_{n-1}\right\}$ is free then $Z$ generates $R$ by Lemma 1 and we are done. Otherwise $\operatorname{Alg}(Z)$ has rank at most $n-2$ and, again by the induction hypothesis, some proper subset $Z^{\prime}$ of $Z$ freely generates $\operatorname{Alg}(Z)$. Reordering the $y_{i}$ 's if necessary we find that $z_{1}=w\left(z_{2}, \ldots, z_{n-1}\right)$. Thus the set $Y^{\prime}$ defined by

$$
y_{1}^{\prime}=y_{1}-w\left(y_{n}, \ldots, y_{n-1}\right), \quad y_{2}^{\prime}=y_{2}, \ldots, y_{n-1}^{\prime}=y_{n-1}
$$

is still a set of generators for $R$, and has degree smaller than the degree of $Y$. This contradiction shows that $Z$ is indeed a free set of generators for $R$.

Let now $H_{k}=\left\{h_{i} \mid d\left(h_{i}\right)=k\right\}$ and $Z_{k}=\left\{z_{i} \mid d\left(z_{i}\right)=k\right\}$. By Lemma 3, both $H_{1}$ and $Z_{1}$ span $R_{1} \bmod R_{0}$. Since $Z_{1}$ is free, $Z_{1}$ is a basis for $R_{1}$ modulo $R_{0}$. Extract a basis $H_{1}^{\prime}$ for $R_{1} \bmod R_{0}$ out of $H_{1}$. Then $Z_{1}$ and $H_{1}^{\prime}$ generate the same subalgebra and, since they have the same finite cardinal, $H_{1}^{\prime}$ is free by Proposition 1. It then follows that $\left\{H_{1}^{\prime} \cup \bigcup_{i \geqq 2} Z_{i}\right\}$ again freely generates $R$.

Assume then, for an induction that, for $i \leqq k$, we have extracted bases $H_{i}^{\prime}$ for $R_{i} \bmod R_{i}^{\prime}$ out of $H_{i}$ in such a way that $\left\{\bigcup_{i=1}^{k} H_{i}^{\prime} \cup \bigcup_{i \geqq k+1} Y_{i}\right\}$ freely generates $R$. By Lemma 3, $Z_{k+1}$ and $H_{k+1}$ both span $R_{k+1} \bmod R_{k+1}^{\prime} . Z_{k+1}$ is actually a basis for $R_{k+1} \bmod R_{k+1}^{\prime}$ since, again by Lemma 3, $R_{k+1}^{\prime} \subset \operatorname{Alg}\left(R_{k}\right)=\operatorname{Alg}\left(Z_{1} \cup \cdots \cup Z_{k}\right)$ and $Z$ is free. Let $H_{k+1}^{\prime} \subset H_{k+1}$ be a basis for $R_{k+1} \bmod R_{k+1}^{\prime}$. Thus, if $z \in Z_{k+1}$, then

$$
z=\sum \beta_{j} h_{j}+u, \quad \beta_{j} \in \phi, h_{j} \in H_{k+1}^{\prime}, u \in R_{k+1}^{\prime}
$$

and, noting that by Lemma $3 R_{k+1}^{\prime} \subset \operatorname{Alg}\left(\bigcup_{i=1}^{k} H_{i}^{\prime}\right)$, we find that $Z_{k+1} \subset$ $\operatorname{Alg}\left(\bigcup_{i=1}^{k+1} H_{i}^{\prime}\right)$. It then follows that the set $\left\{\bigcup_{i=1}^{k+1} H_{i}^{\prime} \cup \bigcup_{i \geq k+2} Z_{i}\right\}$ generates $R$. However it must freely generate $R$ since it has the same cardinal as $Z$.

Since this replacement process eventually replaces $Z$ by a subset of $H$, the theorem is proved.

2. We now prove our main theorem

THEOREM 2. Let $\mathfrak{v}$ be a variety in which finitely generated homogeneous subalgebras of free $\mathfrak{v}$-algebras are again free $\mathfrak{v}$-algebras. Let $Y=\left\{y_{1}, \ldots, y_{n}\right\}$ be elements of $F$. Then there is a sequence of degree reducing elementary transformations of order $n$ such that the elements of positive degree in $\left(\cdots\left(Y \tau_{1}\right) \cdots\right) \tau_{k}$ are a free generating set for $\operatorname{Alg}(Y)$.

In particular a Schreier variety is a Nielsen variety.

Proof. Clearly we need only show that if $Y$ contains elements of positive degree and these elements are not a free set, then we can reduce the degree of $Y$ by an elementary transformation. We assume, for convenience, that $Y$ consists of elements of positive degree, and we write $y_{i}=\bar{y}_{i}+h_{i}$ with $d\left(h_{i}\right)=d\left(y_{i}\right), \bar{y}_{i}=0$ or $d\left(\bar{y}_{i}\right)<d\left(h_{i}\right)$ and $h_{i}$ homogeneous. Let $H=\left\{h_{1}, \ldots, h_{n}\right\}$. We first show that if $H$ is 
free, then so is $Y$. For let $0 \neq w\left(x_{1}, \ldots, x_{n}\right)$ be an element of minimal length for which $w\left(y_{1}, \ldots, y_{n}\right)=0$. As in Lemma 2, we write

$$
w\left(x_{1}, \ldots, x_{n}\right)=\sum_{i \in S_{1}} \alpha_{i} m_{i}\left(x_{1}, \ldots, x_{n}\right)+\sum_{i \in S_{2}} \alpha_{i} m_{i}\left(x_{1}, \ldots, x_{n}\right) ; \quad \alpha_{i} \in \phi, m_{i} \in B
$$

where $m_{i}\left(h_{1}, \ldots, h_{n}\right) \neq 0, i \in S_{1}$ if $d\left(m_{i}\left(h_{1}, \ldots, h_{n}\right)\right)=\max _{i}\left(d\left(m_{i}\left(h_{1}, \ldots, h_{n}\right)\right)\right)$ and $i \in S_{2}$ otherwise. Thus, since $w\left(y_{1}, \ldots, y_{n}\right)=0$, we must have $\sum_{i \in S_{1}} \alpha_{i} m_{i}\left(h_{1}, \ldots, h_{n}\right)$ $=0$ and, since $H$ is free, $\sum_{i \in S_{1}} \alpha_{i} m_{i}\left(x_{1}, \ldots, x_{n}\right)=0$. Thus, if $S_{2}=\varnothing$, then $w\left(x_{1}, \ldots, x_{n}\right)=0$ and if $S_{2} \neq \varnothing$, then

$$
0=w^{\prime}\left(y_{1}, \ldots, y_{n}\right)=\sum_{i \in S_{1}} \alpha_{i} m_{i}\left(y_{1}, \ldots, y_{n}\right) \text {. }
$$

However, $w^{\prime}$ is then shorter than $w$ so we have a contradiction in both cases. Thus, if $H$ is free there is nothing to prove.

If $H$ is not free, then, by Theorem 1, some proper subset $K \subset H$ freely generates $\operatorname{Alg}(H)$. Thus, reordering the $y_{i}$ 's if necessary, we find that $h_{1}=w\left(h_{2}, \ldots, h_{n}\right)$ for some $w\left(x_{2}, \ldots, x_{n}\right) \in F$. Define $\tau$ by setting $\left(x_{1}, \ldots, x_{n}\right) \tau=\left(x_{1}, \ldots, x_{n-1}, x_{n}-\right.$ $\left.w\left(x_{2}, \ldots, x_{n}\right)\right)$. Then $Y \tau$ still generates $\operatorname{Alg}(Y)$, and has smaller degree than $Y$. This proves Theorem 2.

IV. Nielsen varieties are Schreier varieties., It now follows easily from Theorem 2 that if finitely generated homogeneous subalgebras of free $\mathfrak{v}$-algebras are free, then all subalgebras of free $\mathfrak{v}$-algebras are free:

THEOREM 3. If $\mathfrak{v}$ satisfies the hypotheses of Theorem 2 , then $\mathfrak{v}$ is a Schreier variety. Thus a Nielsen variety is a Schreier variety.

Proof. Let $F$ be a free algebra in $\mathfrak{v}$, and let $R$ be a subalgebra of $F$. Let $Y_{k}$, for $k \geqq 1$, be a basis for $R_{k} \bmod R_{k}^{\prime}$. It is clear that $Y=\bigcup_{i=1}^{\infty} Y_{i}$ generates $R$. We show that $Y$ freely generates $R$. For suppose not. Then there is a finite subset $\bar{Y}=$ $\left\{y_{1}, \ldots, y_{k}\right\}$ of $Y$ which is not free. Since $\mathfrak{v}$ satisfies the hypotheses of Theorem 2, we find, as in the proof of that theorem, that we may reorder $\bar{Y}$ if necessary in such a way that there is an element $w\left(y_{i_{1}}, \ldots, y_{i_{k}}\right)$ of $F$ such that

$$
d\left(y_{1}-w\left(y_{i_{1}}, \ldots, y_{i_{k}}\right)\right)<d\left(y_{1}\right)
$$

with $d\left(y_{i_{j}}\right) \leqq d\left(y_{1}\right)$ and $i_{j} \neq 1$. Thus the terms of degree $d\left(y_{1}\right)$ in the left hand side of (2) must vanish. It is now easy to see that (2) contradicts the fact that the $y_{i}$ 's of degree $d\left(y_{1}\right)$ are $\phi$-independent modulo $R_{d\left(y_{1}\right)}$.

\section{Applications.}

1. Following the proofs in [6, Theorem 3.2] and [2, Theorem 6.3] it is now an easy matter to obtain

THEOREM 4. Let $F=F\left(x_{1}, \ldots, x_{n}\right)$ be the free algebra of rank $n$ in a Schreier variety. The group of automorphisms of $F$ is generated by the permutations of $x_{1}, \ldots, x_{n}$ together with the automorphisms

$$
\begin{aligned}
& \alpha_{\varphi}: x_{1} \rightarrow \varphi x_{1} ; \quad x_{i} \rightarrow x_{i}, \quad(i \neq 1)(\varphi \in \phi, \varphi \neq 0), \\
& \tau_{w}: x_{1} \rightarrow x_{1}+w\left(x_{2}, \ldots, x_{k}\right), \quad x_{i} \rightarrow x_{i}, \quad(i \neq 1) .
\end{aligned}
$$


Likewise, following the proof in [6, Theorem 3.3, p. 132] we obtain

Theorem 5. Let $F$ be as above, and suppose $N$ is an ideal of $F$ such that $F / N$ is again a free $\mathfrak{v}$-algebra. Then there exists a set of generators $y_{1}, \ldots, y_{n}$ of $F$ such that $N$ is the ideal generated by $y_{1}, \ldots, y_{k}(k \leqq n)$.

2. We now turn our attention to word problems. Specifically, let $F=F\left(\left\{x_{1}, \ldots, x_{n}\right\}\right)$ be the (absolutely) free algebra freely generated by $x_{1}, \ldots, x_{n}$, let $N$ be an ideal of $F$, and let $A=F / N$. We say that we can solve the word problem in $A$ if, given an element $w \in F$ there is an integer $f(w)$ which depends only on the degree of $w$ such that we can decide in at most $f(w)$ steps whether $w \in N$ or $w \notin N$. It is clear that the word problem is only meaningful if the field $\phi$ is given in an effective manner, and we will assume this in the remainder of this paper. We shall use the fact that in this case it follows from elementary linear algebra that if $E$ is a $k_{1} \times k_{2}$ system of linear equations over $\phi$, then there is an integer $e\left(k_{1}, k_{2}\right)$ such that, in at most $e\left(k_{1}, k_{2}\right)$ steps, we can find a solution to $E$ or decide that $E$ has no solution.

Many of the details in the following proofs are easy but cumbersome and will be omitted.

Lemma 3. Suppose that $N$ has a set $G$ of homogeneous ideal generators such that, for any $k, G_{k}=\{g \in G \mid d(g)=k\}$ is finite and can be effectively enumerated. Then we can solve the word problem in $A$.

Proof. Define inductively

$$
\begin{aligned}
& G^{0}=G, \\
& G^{i}=\left\{l(g r) \mid l, r \text { monomials, } g \in G^{i-1}\right\} \cup\left\{(l g) r \mid l, r \text { monomials, } g \in G^{i-1}\right\}
\end{aligned}
$$

and let $\bar{G}=\bigcup_{i=0}^{\infty} G^{i}$. Then $\langle\bar{G}\rangle=N$. Further, since $F$ is finitely generated, $F$ has only finitely many monomials of a given degree and hence $\bar{G}_{k}=\{\bar{g} \in \bar{G} \mid d(\bar{g})=k\}$ is still finite and can be effectively enumerated. In fact we can enumerate $\bar{G}_{k}$ in $r(k)$ steps, where $r$ is an integer which only depends on $\phi, G, n$ and $k$.

Let $w \in F$. Then $w \in N$ if and only if the homogeneous terms of $w$ all lie in $N$ and we may thus assume that $w$ is homogeneous, say of degree $k$. If $w \in N$, then

$$
w=\sum \beta_{i} \bar{g}_{i}, \quad \beta_{i} \in \phi, \bar{g}_{i} \in \bar{G},
$$

and, since each $\bar{g}_{i}$ is homogeneous, we may assume that

$$
w=\sum \beta_{i} \bar{g}_{i}, \quad \beta_{i} \in \phi ; \bar{g}_{i} \in \bar{G}_{k} .
$$

We now write $w=\sum \alpha_{s} m_{s}$ and $\bar{g}_{i}=\sum \alpha_{i, s} m_{s}$, where $m_{s}$ ranges over the monomials of degree $k$. (3) now gives rise to a finite system $E$ of linear equations and $w \in N$ if and only if $E$ has a solution. Thus the proof is complete. We note that if $w \in N$ this method gives an expression for $w$ in terms of the generators $G$ of $N$. 
Lemma 4. If $N$ is as in Lemma 3 and $N \neq F$, then we can find a set $B=\left\{b_{0}=1\right.$, $\left.b_{1}, \ldots\right\}$ of monomials of $F$ with the properties:

(i) $\left\{b_{i}+N\right\}, i=0,1, \ldots$, is $a \phi$-basis for $A$.

(ii) If $w \in F$ is of degree $k$, then there is an integer $s(k)$ which depends only on $k, G$, $n$ and $\phi$ such that we can find in at most $s(k)$ steps the unique expression

$$
w=\alpha_{1} b_{i_{1}}+\alpha_{2} b_{i_{2}}+\cdots+\alpha_{r} b_{i_{r}}+t, \quad \alpha_{i} \in \phi, b_{i_{1}} \in B, t \in N .
$$

Proof. We order the monomials of $F$ lexicographically and declare 1 to be smaller than any other monomial. We define $B$ inductively by setting $B^{0}=\{1\}$ and defining $B^{i}=B^{i-1} \cup\left\{b_{i}\right\}$ if $b_{i}$ is the least monomial in the order such that $B^{i} \cup\left\{b_{i}\right\}$ is still linearly independent modulo $N$ or defining $B^{i}=B^{i-1}$ if there is no such monomial. We then set $B=\bigcup_{i=0}^{\infty} B^{i}$. It is clear that $B$ has property (i). We now show by induction that we can effectively enumerate $B$. For suppose we can effectively enumerate $B^{k}$, and suppose that the greatest monomial in $B^{k}$ has degree $p$. Let $B_{p}^{k}=\{b \in B \mid d(h)=p\}$. Let $m$ be a monomial of degree $p$ greater than any monomial in $B^{k}$. To test whether $\{m\} \cup B^{k}$ is still independent, we adjoin $B_{p}^{k}$ to $G$ and consider $N^{\prime}=$ Id $\left(G \cup B_{p}^{k}\right)$. By Lemma 3 we can decide if $m \in N^{\prime}$. If $m \notin N^{\prime}$, then we can set $B^{k+1}=B^{k} \cup\{m\}$. If $m \in N^{\prime}$ then, by Lemma 3 , we can effectively express $m$ in terms of $G \cup B_{p}^{k}$ and, since $d(m)=p$, this expression is of the form (4). If no monomial of degree $p$ can be adjoined to $B^{k}$ to define $B^{k+1}$, and some monomial of degree between $p$ and $2 p$ is not in $N$, then we choose $m$ to be the least such monomial and define $B^{k+1}=B^{k} \cup\{m\}$. This, again by Lemma 3 , can be done effectively. Finally, if all the monomials of degree between $p$ and $2 p$ are in $N$, then every monomial of degree $>p$ is in $N$ and we set $B^{k+1}=B^{k}$.

It is now clear from the foregoing that property (ii) is satisfied whenever $w$ is a monomial. Since any element of $F$ is a linear combination of finitely many monomials, (ii) is satisfied for any element of $F$ and the proof is complete.

We remind the reader that a variety $\mathfrak{v}$ is defined via a subset $V$ of the free algebra $F(X)$ of countably infinite rank. If, whenever $v\left(x_{1}, \ldots, x_{r}\right) \in V$ and $w_{1}, \ldots, w_{i}$, $w_{i}^{\prime}, \ldots, w_{r} \in F(X)$, then

$$
v\left(w_{1}, \ldots, w_{i}+w_{i}^{\prime}, \ldots, w_{r}\right)=v\left(w_{1}, \ldots, w_{i}, \ldots, w_{r}\right)+v\left(w_{1}, \ldots, w_{i}^{\prime}, \ldots, w_{r}\right),
$$

we say that $\mathfrak{v}$ is defined by multilinear laws. We note that if $\phi$ has characteristic $p \neq 2$, then the varieties of Lie algebras, commutative algebras, anticommutative algebras can be defined by multilinear laws.

THEOREM 6. Let $\mathfrak{v}$ be a variety defined by finitely many multilinear laws. Then we can solve the word problem in $F\left(\mathfrak{b},\left\{x_{1}, \ldots, x_{n}\right\}\right)$.

Proof. We first note that, by Proposition 2, we may assume that $V$ consists of finitely many homogeneous multilinear elements. It now follows from the multilinearity that $\mathfrak{v}\left(F\left(x_{1}, \ldots, x_{n}\right)\right)$ is generated, qua ideal, by

$$
G=\left\{v\left(m_{1}, \ldots, m_{k}\right) \mid v \in V, m_{1}, \ldots, m_{k} \text { monomials }\right\} .
$$

We can then apply Lemma 3 to complete the proof. 
3. We apply our last results to Schreier varieties. If $\mathfrak{v}$ is a variety defined by finitely many multilinear laws, then, in view of Theorem 6 and Lemma 4, there exists a monomial basis $B$ for $F=F\left(\mathfrak{b},\left\{x_{1}, \ldots, x_{n}\right\}\right)$ such that an expression in the generators of $F$ can be effectively written as a linear combination of the elements of $B$.

THEOREM 7. Let $\mathfrak{v}$ be a Schreier variety defined by finitely many multilinear laws. Then the generalized word problem is solvable in $F=F\left(\mathfrak{b},\left\{x_{1}, \ldots, x_{n}\right\}\right)$, i.e., given elements $z_{1}, \ldots, z_{r}, z$ in $F$, one can decide effectively whether $z \in \operatorname{Alg}\left(z_{1}, \ldots, z_{r}\right)$.

Proof. We first show that if $y_{1}, \ldots, y_{r}$ are elements of $F$ with highest homogeneous terms $h_{1}, \ldots, h_{r}$, then we can effectively find a set $y_{1}^{\prime}, \ldots, y_{s}^{\prime}$ of elements of $F$, with homogeneous terms $h_{1}^{\prime}, \ldots, h_{s}^{\prime}$ such that $y_{1}^{\prime} \ldots, y_{s}^{\prime}$, freely generate $\operatorname{Alg}\left(y_{1}, \ldots, y_{r}\right)$ and $\left\{h_{1}^{\prime}, \ldots, h_{s}^{\prime}\right\}$ is free. For, by Theorem $1,\left\{h_{1}, \ldots, h_{r}\right\}$ is not free only if, after a suitable reordering of $h_{1}, \ldots, h_{r}, h_{1} \in \operatorname{Alg}\left(h_{2}, \ldots, h_{r}\right)$. If $h_{1} \in$ $\operatorname{Alg}\left(h_{2}, \ldots, h_{r}\right)$ then, since $h_{2}, \ldots, h_{r}$ are homogeneous, $h_{1}$ is a linear combination of the products $h_{i_{1}} h_{i_{2}} \cdots h_{i_{k}}$ which have degree $d\left(h_{1}\right)$. By the above remark, we can effectively write each of these products as a linear combination of elements of $B$, and thus, in order to decide if $h_{1} \in \operatorname{Alg}\left(h_{2}, \ldots, h_{r}\right)$, we are led to consider a system of linear equations over $\phi$. If there are no solutions, then $h_{1} \notin \operatorname{Alg}\left(h_{2}, \ldots, h_{r}\right)$. On the other hand a solution defines an elementary transformation which reduces the degree of $Y=\left\{y_{1}, \ldots, y_{r}\right\}$. Thus, to prove our claim we need only apply the above procedure to every reordering of $h_{1}, \ldots, h_{r}$ and use induction on the degree of $Y$.

We may then assume that the highest homogeneous terms $h_{1}, \ldots, h_{r}$ of $z_{1}, \ldots, z_{r}$ form a free set. We prove the theorem by induction on the degree of $z$. If $d(z)=0$, then $y \in \operatorname{Alg}\left(z_{1}, \ldots, z_{r}\right)$ and there is nothing to prove. Suppose then that $d(z)=k$, and that $d\left(h_{1}\right) \leqq d\left(h_{2}\right) \leqq \cdots \leqq d\left(h_{s}\right) \leqq k$ and $d\left(h_{s+i}\right)>k$. Let $h$ be the highest homogeneous term of $z$. Then, using now familiar arguments, we see that if $\left\{h_{1}, \ldots, h_{s}, h\right\}$ is a free set then $z \notin \operatorname{Alg}\left(z_{1}, \ldots, z_{r}\right)$. Thus, proceeding as in the first part of the proof, we find that $z \notin \operatorname{Alg}\left(z_{1}, \ldots, z_{n}\right)$ or we find an element $w\left(z_{1}, \ldots, z_{s}\right)$ such that $d\left(z-w\left(z_{1}, \ldots, z_{s}\right)\right)<k$. However, since $z-w\left(z_{1}, \ldots, z_{s}\right) \in \operatorname{Alg}\left(z_{1}, \ldots, z_{r}\right)$ if and only if $z \in \operatorname{Alg}\left(z_{1}, \ldots, z_{r}\right)$, the proof is complete by induction.

\section{REFERENCES}

1. R. H. Bruck, A survey of binary systems, Ergebnisse der Mathematik und ihrer Grenzgebiete, N. F., Heft 20, Springer-Verlag, Berlin, 1958.

2. P. M. Cohn, Subalgebras of free associative algebras, Proc. London Math. Soc. 56 (1964), 618-632.

3. - Universal algebra, Harper and Row, New York, 1965.

4. N. Jacobson, Structure of rings, rev. ed., Amer. Math. Soc. Colloq. Publ., Vol. 37, Amer. Math. Soc., Providence, R. I., 1964.

5. A. G. Kuroš, Nonassociative free algebras and free products of algebras, Mat. Sb. 20 (62) (1947), 239-262. (Russian) 
6. W. Magnus, A. Karrass and D. Solitar, Combinatorial group theory, Interscience, New York, 1966.

7. J. Nielsen, Die Isomorphismengruppe der freien Gruppen, Math. Ann. 91 (1924), 169-209.

8. O. Schreier, Die Untergruppen der freien Gruppen, Abh. Math. Sem. Univ. Hamburg 5 (1927), 161-183.

9. A. I. Siršov, On subalgebras of free Lie Algebras, Mat. Sb. 33 (75) (1953), 441-453. (Russian)

10. - On subalgebras of free commutative and anticommutative algebras, Mat. Sb. 34 (76) (1954), 81-88. (Russian)

11. E. Witt, Uber freie Ringe und ihre Unterringe, Math. Z. 58 (1953), 113-114.

12. —_, Die Unterringe der freien Lieschen Ringe, 64 (1956), 195-216.

SyRACUSE UNIVERSITY,

Syracuse, New York 